DR. WEIR-The difficulties I have experienced occurred in buttons made by instrument makers who were indorsed by Dr. Murphy, hence the more likely to happen with less expert manufacturers. Dr. Rodman's suggestion is a scientific one and worthy of consideration. There is one point that Dr. Mayo brought out by inference and that is that the procedure is one only to be used where the gastroenterustomy or other procedure has failed. This should be strongly emphasized.

Dr. Rodman - One can complete a pylorectomy in any way that he pleases, either by the first or the second method of Billroth. Personally I should think it better to close it entirely. The principal claim for this operation is the fact that there is a large and unknown per cent. of cases of gastric ulcer which result in malignant change and that this is an increasing per cent. all will admit. It should only be resorted to in cases where it would seem that probably a gastroenterostomy would not prove successful as, for instance, where there were multiple ulcers. In those cases where the ulcer is large and where the tumor is accompanied by glandular involvement, as in a certain per cent. of benign ulcers, so that no one could possibly say that he was dealing with a benign condition, then I say it is certainly a rational procedure, for in such a condition we have to recognize and admit that there is a fair probability at least of malignant disease in men over 50. Every one realizes the fact that chronic ulcer of the stomach so often forms adhesions and takes in the lymphatic glands that it is not possible at the time of the operation to say that it is benign. Kehr and others have removed 1 umors that they supposed to be malignant which proved to be benign. I can not believe that the mortality can possibly be 30 or 40 per cent. It should not be followed by a mortality of more than 15 per cent. Even in malignant disease we realize that pylorectomy has reduced the mortality to less than 30 per cent. It must always be an operation followed by a somewhat heavier mortality than gastroenterostomy. Mayo did not believe it would be followed by a mortality as great as that. Gastroenterostomy has a mortality of 5 or 6 per cent. There is a difference, but it is not very great, and in favorable cases it seems to me that it may have an accepted place in surgery.

\section{BORIC-ACID POISONING.}

REPORT OF A FATAL CASE, WITH AUTOPSY.

\section{CHARLES L. BEST, M.S.}

CHICAGO.

From the Pathological Laboratory of the University of Chicago.

The opinion appears to exist that boric acid, even in large amounts, is entirely innocuous to the human body when applied externally to wounds, suppurating cavities, etc. That this is erroneous and that the surgeon must be on his guard against its too free usage, as it is not impossible for a fatal issue to ensue, is shown by the literature, in which five cases of severe intoxication and four deaths are reported in addition to the case at hand. These cases all resulted from the prolonged irrigation with saturated solutions of boric acid or the packing of large cavities with the dry powder.

Welch $^{\mathbf{1}}$ reports his experience in the treatment of lencorrhea, in which he packed the upper third of the ragina with boric acid. allowing it to remain until liquefied, usually two to three days. This was repeated every secenth day. In three cases intoxication resulted, and are reported by him as follows:

CASE 1.- Two hours after the first treatment the patient was found with the skin cool, pulse feeble, respiration weak, eyes sunken and dull and the mind unclouded but despondent.

CASE 2.-Two days after the seventh treatment there was a marked depression of the nervous system and a pronounced acid discharge from the vagina. The skin of the hands, face and feet was swollen, became charred and finally exfoliated. All motion was very painful.
Case 3.- Wwo days after the seventh treatmenc the patieut was found in a state of collapse, low spirited, the puse reebre, the eyes sunken, the face dusin, and general weakness. There was great pain in the vagina, the mucosa of which was corroded, and much discharge from the part. Temperature, 97.6; pulse, 60. The symptoms did not subside for a week, in the meantime the patient being at times cold, hysterical and prone to melancholy. The skin exfoliated in branny scales. All three cases recovered.

Spencer, a atter an operation for syphilitic necrosis of the ankle joint packed the cavity with one-half ounce of boric acid. 'l'liis was repeated on the seventh day, and on the fourtcenth nausea and vomiting set in, not yieıding to treatment. Other symptoms were restlessness, insomnia, hiccough, weakness, emaciation, rapid pulse, some coryza, inflammation of fauces and pharynx, bronchitis, an acid discharge from the anterior nares and a well-marked papular erythema over the face, neck, arms and chest. The symptoms came on gradually, increasing for three days, when the dressings were changed, and in ten days they had disappeared.

In addition, he speaks of two cases, one in washing out a lumbar abscess, and the other the pleural cavity, with a 5 per cent. solution of boric acid, followed by death in both cases, the symptoms being nausea, vomiting, hiccough, slight temperature, bronchial catarrh, a weak, rapid pulse and well-marked papular erythema. The report of these I was unable to find.

Hun," after irrigating the pleural cavity with 16 ounces of a saturated solution of boric acid, found his patient in the evening covered with a papular erythema, and with a rapid, soft pulse. A sccond irrigation on the following day aggravated these symptoms, and nausea and vomiting set in. The treatment was discontinued and the patient recovered.

Molodenow ${ }^{4}$ used a 5 per cent. solution very freely for washing out the pleural cavity in one patient and a lumbar abscess in another. This treatment was continued for one hour. Uncontrollahle vomiting set in in both cases, followed by an erythema of the face and neck, and death from cardiac paralysis. Autopsy was not secured.

Williams ${ }^{5}$ irrigated freely a case of empyema, the treatment extending over a period of two months, and finally placed 5 ounces of the dry powder in the cavity. Delirium set in, shortly followed by uncontrollable vomiting, an erythema over the body, cardiac paralysis and death. Autopsy was not secured.

Rose $^{6}$ fully reports a case, which may be summarized as follows :

History.-Boric acid was freely used in the treatment of a large open sore on the right thigh. On the fifth day a profuse diarrhea set in, but there was no change in the general condition. The mind was clear, temperature normal, with pulsation slightly increased. Later vomiting set in and the granulations assumed a sluggish appearance. The boric acid was discontinued. The diarrhea and vomiting, which consisted chiefly of mucus and water, became uncontrollable. On the ninth day the intellect was still clear, but the eyes staring, the body was batherl with a cold, clammy perspiration, and there was great weakness. The hiccoughing and the vomiting were but partially relieved by morphia. The urine for the last few days was intensely acid. The temperature was 97.4 ; pulse, 105. Death occurred on the following day, the temperature being 96 , the pulse 140. Just before death the tongue was red and moist, the vomiting almost continuous, and the urine al. most completely suppressed.

2. Northwestern Jancet, 1888 , vol. i, p. 22.

3. Medical News. Philadelphia, 1882, vol. x1, p. 704

4. Tillman's Operative Surgery.

5. Unreported case of V. Williams, Chicago.

6. Medical News, Philadelphia, 1883, vol. xliii, p. 199. 
Autopsy.-There were found congestion of the liver, spleen and gastrointestinal tract, with several erosions in the mucosa of the stomach. Histologically, the liver showed cloudy swelling, granular degeneration and connective tissue proliferation. The kidney tubules contained casts and the cells were swollen and granular. Much pigment was found both in the liver and kidney.

Summarizing these cases, it will be found that nausea and uncontrollable vomiting were present except in Welch's cases, all of which recovered, and all being cases in which the application was made to the vaginal mucosa, from which we may assume some difference in the absorption between this and ordinary raw surfaces. The same is true of the papular erythema of the face, neck and chest, with the additional exception of Rose's case. Depression of the nerrous system and general muscular weakness is constant. In the two cases in which the temperature was recorded it was subnormal throughout. A cold, clammy perspiration was present in one-half the cases. The pulse was feeble, but of normal frequency, except in the fatal cases, where it gradually inereased before death. An inflammatory condition of the pharynx, larynx and nares was confined chiefly to those cases in which there had been irrigation of the pleural cavity. Delirium was present in but one case, and that just before death. The postmortem and histologic findings in the one case that came to autopsy were those of an acute intoxication The onset varied from twelve hours to seven days, usually beginning with profuse vomiting, followed in the comrse of a day by an erythema, weak pulse, depression of the nervous system and general bodily weakness.

To these cases, which are all that can be found in the literature, I desire to add the report of a fatal case which I have had the opportunity to study in the laboratory of pathology of the University of Chicago:

\section{REPORT OF A CASE.}

Patient.-W. A. D., aged 36, occupation salesman, was admitted to hospital Aug. 16, 1903, with the following history: Previous to the present trouble he had been quite well, He complained of pain in the right inguinal region, and stated that about five months previously he had noticed a swelling in this region which was diagnused inguinal hernia. A truss was worn for four and one-half months, but the condition gradually grew worse. Suppurative lymphadenitis was then diagnosed. At this time the right ear showed signs of inflammation, and paracentesis was performed.

Examination.-The physical examination at the hospital showed both ears tender on pressure, the hearing impaired, the membranes inflamed and the right mastoid region swollen, red and painful. In the right inguinal region just above Poupart's ligament was found a large swelling, with marked fluctuation, painful on pressure and very red. Otherwise the examination was negative.

Diagnosis.-Suppurative inguinal lymphadenitis, bilatera] otitis media, right suppurative mastoiditis.

Operation.--The following day the right inguinal glands were completely excised, and much pus was found. The wound was washed out with salt solution, packed with approximately if oz. of boric acid powder, sewed up without drainage, and realed with collodion. A large opening was made in the right trmpanic membrane at the same time. After the operation there was marked emesis of a greenish yellow fluid, which coninued and became uncontrollable before death.

Course-On the evening of the third day a diffuse erythematous and slightly papular rash appeared on the neck, chest and shoulders, with a distinet line of demarcation at the clavicle. The following day a more diffuse papular rash appeared "n the bark and thighs. There was no pustule formation. The skin about the operation wound was red. On August 20, 1 a. m., there was marked cyanosis, a clammy sweat, cold feet, weak, irregular pulse and uncontrollable vomiting. Delirium set in during the last few hours before death. The patient died at 7 a. m., Aug. 20, 1903. The rash was still visible. The temperature had increased with slight remissions from 98.4 on admis sion to 100.8 , with a fall of 0.6 just before death. The pulse increased from 68 on admission to 138 , with a decrease of 12 just before death. Respiration on admission was 16 , which in creased to 38 just prior to death.

Autopsy.-An autopsy was held three hours later by Dr. $\mathrm{H}$. G. Wells. The body was that of a well-developed, well-nourished man, $165 \mathrm{~cm}$. in length, not yet cold, but rigor mortis was present. The skin was white except for a purplish tinge above the clavicle and on the right side of the scrotum, and a brownish discoloration about the inguinal wound where the epidermis was elevated by a large quantity of serous fluid, which readily escaped, leaving the epidermis unattached. The lymph glands were not enlarged.

The operation wound, $11 \mathrm{~cm}$ long, extended to the abdominal muscles and was filled with a chocolate-colored grumous ma terial. No pus was present in the wound, but beneath the peri toneum, posterior to the right rectus and extending under Poupart's ligament along the sheath of the great vessels was a cavity filled with a creamy pus from which several sinuses extended-one $5 \mathrm{~cm}$. in length along the external border of the right rectus to the floor of the operation wound, but separated from it by a layer of fascia $2 \mathrm{~mm}$. thick, and the other along the inner side of the ramus of the ischium. The cavity was lined by a soft pinkish tissue, in the floor of which was seen the internal iliac artery, but neither this nor any other of the vessels were thrombosed. The regional lymph glands were enlarged, the mesenteric normal in size and consistency. The peritoneal cavity was free from adhesions or fluid.

The left pleural cavity contained many fibrous adhesions posteriorly, and the right was completely obliterated by the same and showed a few pinhead-sized points of calcification on the pleura. The visceral pericardium showed innumerable minute subpericardial extravasations of blood, from which the parietal layer was free.

The musculature of the heart was normal, likewise the valves, and the vessels were free from sclerosis. A large postmortem blood clot was found in the right ventricle. The blood in the heart was mostly unclotted, of a granular, turbid appearance. with a slight magenta tinge.

The lungs showed no areas of consolidation, crepitation be ing present throughout. A large amount of blood and frothy fluid exuded on handling. The peribronchial glands were nor. mal but for a few areas of calcification.

The gall bladder was distended, containing a dark, tarry bile, but no cal'uli were present. The liver was enlarged, weight 2,500 gnis., pale and friable, and on the cut surface only the centers of the lobules appeared normal, the peripheries being pale.

About the spleen there were a few fibrous adhesions. Its weight was $250 \mathrm{gms}$. On the cut surface much blood was seen. though the general consistency was about normal.

The adrenals showed no change. The kidneys showed a thick, fatty capsule, were of normal size and consistence and showed remnants of fetal lobulation. The cortex was pale and the markings fairly distinct. The capsule stripped readily, leaving a smooth surface.

The surfaces of the gastrointestinal tract were normal; the prostate showed no changes.

The cranial cavity showed a moderate amount of fluid, the longitudinal sinus containing a soft, dark clot, not adherent. The brain substance was normal. No change in meninges, skull nor the adjacent sinuses.

Anatomic Diagnosis.-Recent operation wound in right inguinal region; suppurating sinuses, subperitoneal and about the sheaths of the right iliac and femoral vessels; postmortem wound over right mastoid; bilateral obliterative pleuritis; healed calcified tubercles of peribronchial glands and pleura; fatty changes in liver and kidney; fibrous cholecystitis; hyper plasia of vermiform appendix; subpericardial ecchymoses.

Bacteriologic Findings.-From the heart's blood: A smear was negative. No growths obtained on culture, except one 
tube contaminated by bacillus subtilis. From the wound in the inguinal region the staphylococcus albus was obtained.

All cultures from the different viscera remained sterile.

Histology.-Skin from the neck (Erythema). Desquamation of the stratum corneum down to the stratum lucidum; much hemorrhage into this portion of the epidermis and the gland crypts; much round and epithelioid cell invasion of the stratum malpighii; comparatively little leucocytic invasion; distension of vessels of the musculature with blood.

The spleen showed some congestion; no other changes.

The lungs showed extensive destruction of air cells, congestion of large and small blood vessels and deposition of coal pigment, especially about the larger vessels. The air cells in many places were filled with red and white blood corpuscles. Polymorphonuclear leucocytes were numerous. A small amount of fibrin was presen $i$ in a few alveoli. No bacteria were shown by polychrome methylene blue staining.

The lumen of the appendix was filled with areolar tissue traversed by fibrous bands, showing numerous round lymphoid cells at one point.

In the liver there was considerable fatty infiltration and some round cell proliferation in the interlobular septa about the ducts, but no other changes.

The vessels of the capsule and of the kidney itself were much rongested; otherwise no changes.

The heart muscle showed slight increase in connective tissue, and some pigmentation of the muscle fibers.

The pancreas was normal.

The prostate showed a glandular proliferation of the epithelium and the acini filled with a hemorrhagic exudate and granular débris.

The abscess wall showed the musele fibers scattered and a marked increase in connective tissue, also an infiltration of round cells and many red and white blood corpuscles, the latter chiefly of the polymorphous variety.

An analysis of a sample of the boric acid used in the hospital, and from which that used in this case was taken, was made, and the chemist reported it to be rhemically pure.

Unfortunately, the reactions of the blood and secretions were not taken.

The lack of other causes, and the fact that clinically this case co closely resembles those previously mentioned, leaves no doubt as to the identity, all the cardinal points of boric-acid intoxication being present: profuse vomiting, a papular rash over the face, neck and chest, and a weak irregular pulse increasing before death. It difters only in its more rapid onset and course, the onset being immediate and the fatal termination in four days. The delirium and the slight rise in temperature are possibly indications of a greater degree of intoxication.

'The postmortem and histologic findings in lose's case are identical with those found here, the former being entirely nogative except for a congestion of the liver, spleen and gastro-intestinal tract and a cloudy swelling and granular degeneration of the liver and kidney cells. In tlie latter we have practically the same findings, with the addition of subpericardial hemorrhages and fatty changes in the liver and kidney, together with discoloration and maceration of the tissues about the cavity in which the boric acid was placed.

These findings then limit the cause of death to one of two things: a toxemia, resulting either from the abscess in the groin or from the medicinal agent employed, as other recognizable causes were not present. Its resemblance to those cases previously reported, toyether with the demonstrated overuse of this substance, is sufficient to make a positive diagnosis of fatal boricacid intoxication. The clinical features and the lack of success in cultivating pathogenic bacteria from the blood, eliminate septicemia in this case, which is the condition most likely to simulate such an intoxication.

\section{A METHOD OF DISPENSING WITH RUBBER GLOVES AND THE ADHESIVE RUBBER DAM.}

\author{
SECOND COMMUNICATION.
}

JOHN B. MURPHY, A.M., M.D.

Professor of Surgery, Northwestern University Medical School: Attending Surgeon, Mercy Hospital, Cook County Hospital.

\section{Chicago.}

A short time ago ${ }^{1}$ I gave a preliminary account of the use of an impermeable coating designed to prevent the escape of the epithelium and sebaceous gland secretions from tlie shin during operation, and the entrance of blood, pus, etc., into the crevices of the skin. It consists, briefly, in the application of a 4 to 8 per cent. solution of gutta-percha in benzine or acetone-the benzine solution for use on the hands, the acetone for surface applications at and around the field of operation. The 4 per cent. benzine solution has proven the best for wear. The benzine solution, however, dries more slowly than the acetone; it requires one and a half to two and a half minutes for it to thoroughly evaporate. If the rubber has not been destroyed by heat or sterilizing processes, the parts will have a decided adhesive tendency. If the solution is not good the adhesiveness will not be manifest. The acetone solution evaporates much more rapidly and, therefore, malies the best for the field of operation.

The following experiments show the comparative results between the rubber solution and the gloves, and while great differences in various operations appear, they are due to many extraneous conditions, which are not under control by any means so far suggested. However, they give a fair idea of the comparative advantages of the rubber gloves and solution over the naked hands.

\section{METHOD OF TESTING VALUE OF PROCEDURE.}

The method employed to test the efficacy of the rubber solution in protecting the patient from infection at the hands of the operator was as follows: The soiution was used either by the operator or his assistant, while the other wore gloves or used bare hands. The hands of each were subjected to the same process of disinfection so far as possible. After the operation, each would wash his hands in 1 liter of controlled sterile water. Agar plates were then made with 1 c.c. of the water, and a comparative count of the colonies made in forty-eight to seventy-two hours.

In order to obtain a fair and unbiased result, the following requirements must be fulfilled:

1. Ali chances of contamination from the patient himself must be avoided. Hence all operations on infected cases, on the gastrointestinal tract or biliary passages and in the vicinity of the mucous outlets, were unavailable. As a result, only a limited number of clean operations were left for selection, such, for example, as those for hernia, for varicose veins, for specific disease of the tubes, certain abdominal and somatic tumors, etc.

2. The operator and his assistant should do as ncarly an equal amount of work as is compatible with the operation, so that each would be subject to equal chances of contamination from the patient.

3. Extreme care must be taken not to allow puncture of the gloves to pass unnoticed, as this occurs much more frequently than is generally supposed.

4. The rubber solution, sterile water, etc., should be 